\title{
Collaborative Staging
}

National Cancer Institute

\section{Source}

National Cancer Institute. Collaborative Staging. NCI Thesaurus. Code C62575.

A coding system for staging of cancer bringing together the principles of Summary Stage, the TNM categ ories and stage groupings, and the SEER Extent of Disease (EOD) coding structure, creating a carefully selected set of data items that describe how far a cancer has spread at the time of diagnosis. The Collaborative Staging System uses a modified EOD coding system, a five-field, 10 digit system: tumor size (3 digits), extension of the primary tumor (2 digits), regional lymph node involvement (highest specific lymph node chain involved by tumor) (1 digit), the number of pathologically reviewed regional lymph nodes that are positive (2 digits), and the number of pathologically examined regional lymph nodes (2 digits). 